\title{
Auftreten von Ulcera crurum in Zusammenhang mit der Einnahme von Anagrelid ${ }^{*}$
}

\section{Leg Ulcers Associated with Intake of Anagrelide}

Autoren

Institut

\section{Rappoport, A. Körber, S. Grabbe, J. Dissemond}

Dermatologische Klinik und Poliklinik, Universitätsklinikum Essen

\section{Bibliografie}

DOI $10.1055 / \mathrm{s}-2007-995717$

Akt Dermatol 2008; 34:

132-134 @ Georg Thieme

Verlag KG Stuttgart · New York ISSN 0340-2541

Korrespondenzadresse

Priv.-Doz. Dr. med.

Joachim Dissemond

Universitätsklinikum Essen,

Klinik und Poliklinik für

Dermatologie, Venerologie

und Allergologie

Hufelandstr. 55

45122 Essen

joachimdissemond@

hotmail.com

\section{Zusammenfassung \\ $\nabla$}

Anamnese und klinischer Befund: Wir berichten über einen 38-jährigen Patienten mit äußerst schmerzhaften Ulcera crurum an beiden Außenknöcheln, die 6 Wochen nach Beginn einer Therapie mit Anagrelid bei Thrombozythämie erstmalig auftraten.

Diagnostik: Im Rahmen einer umfassenden Diagnostik (Laboruntersuchungen, Doppler- und Duplex-Untersuchungen der oberflächlichen und tiefen Beinvenen, bakteriologische und mykologische Diagnostik, histopathologische Untersuchung) konnten wir eine vaskuläre, vaskulitische sowie eine metabolische Genese der Ulzerationen ausschließen.

\section{Einleitung \\ $\nabla$}

In Deutschland leiden etwa 1,0-1,5 Millionen Menschen unter einem Ulcus cruris unterschiedlicher Genese. Die häufigste Ursache ist die chronische venöse Insuffizienz (CVI) bei ca. $80 \%$ der Patienten [1]. Neben beispielsweise einer peripheren arteriellen Verschlusskrankheit (pAVK), Vaskulitiden oder Diabetes mellitus kann auch die Einnahme verschiedener Medikamente nicht nur die Abheilung bereits bestehender Wunden behindern, sondern auch die Entstehung eines Ulcus cruris primär verursachen [2] ( $\bullet$ Tab. 1). Wir beschreiben erstmalig das Auftreten von Ulcera crurum nach Einnahme von Anagrelid.
Erstveröffentlichung in: Dtsch Med Wochenschr 2007; 132: 319-321.
Therapie und Verlauf: Eine vollständige Abheilung der initial therapierefraktären Ulzerationen gelang trotz intensivierter moderner Wundtherapie erst nach Umstellung der Medikation von Anagrelid auf Hydroxyurea.

Folgerung: Wir sehen bei dem hier vorgestellten Patienten die Einnahme von Anagrelid als kausal verantwortlich für die Entstehung der Ulcera crurum, obgleich diese mögliche Nebenwirkung in der Literatur erst einmal beschrieben wurde. Bei Ulcera crurum unklarer Genese sind auch unerwünschte Nebenwirkungen von Medikamenten wie beispielsweise Anagrelid in Betracht zu ziehen.

\section{Kasuistik \\ $\nabla$ \\ Anamnese}

Ein 38-jähriger Patient, bei dem nach einem Myokardinfarkt eine essenzielle Thrombozythämie diagnostiziert worden war, erhielt für $3 \mathrm{Jah}$ re $1 \times 500 \mathrm{mg} / \mathrm{d}$ Hydroxyurea. Bereits 6 Wochen nach Umstellung der Medikation auf $2 \times 500$ $\mathrm{mg} / \mathrm{d}$ Anagrelid traten spontan äußerst schmerzhafte Erytheme im Bereich beider Malleoli laterales auf, die im weiteren Verlauf exulzerierten. Als weitere Medikation nahm er ASS, Allopurinol, Lisinopril und Bisoprolol jeweils unverändert seit 3 Jahren ein.

\section{Körperlicher Untersuchungsbefund}

Bei der Erstvorstellung in unserer Klinik war der Patient in einem guten Allgemein- und Ernährungszustand. Die Körpertemperatur war normwertig. Beidseits im Bereich der Malleoli laterales bestanden druckdolente Ulzerationen von $2,0 \mathrm{~cm}$ im Durchmesser rechts sowie $1,5 \mathrm{~cm}$ links. Am rechten Knöchel war das Ulcus nekrotisch belegt und hatte am Wundrand ein ausgeprägtes livides 
Tab. 1 Potenzielle Ursachen von Ulcera crurum.

Gefäßerkrankungen, z.B.CVI, pAVK

Vaskulitiden, z. B. leukozytoklastische Vaskulitis, Polyarteriitis nodosa Neuropathische Erkrankungen, z. B. Diabetes mellitus, Alkohol Metabolische Defekte, z. B. Diabetes mellitus, Kalziphylaxie

Medikamente, z. B. Hydroxyurea, Phenprocoumon

Genetische Defekte, z. B. Klinefelter-Syndrom,

Faktor-V-Leiden-Mutation

Hämatologische Defekte, z. B. Sichelzellanämie, Dysproteinämie

Exogene Faktoren, $z$ B. thermisch, mechanisch

Neoplasien, z. B. Basalzellkarzinom, kutanes Lymphom

Infektionen, z. B. Mykobacteriose, Sporotrichiose

Dermatosen, z. B. Pyoderma gangraenosum, Necrobiosis lipoidica

Erythem, das bis zum distalen Unterschenkeldrittel reichte ( $\bigcirc$ Abb. 1 a). Linksseitig sah man ein fibrinös belegtes Ulcus cruris mit erythematösem Randsaum und Atrophie blanche in der unmittelbaren Umgebung ( $\bullet$ Abb. 1 b).

\section{Klinisch chemische Untersuchungen}

Die serologischen Befunde zeigten keine systemischen Infektzeichen: CRP, BSG und Leukozyten waren ebenso wie das restlich untersuchte Routinelabor inklusive Elektrolyte, Leber- und Nierenwerte normwertig. Auch die antinukleären Antikörper, extrahierbare nukleäre Antikörper, antinukleäre zytoplasmatische Antikörper, HbA1c, Kryoglobuline, Antiphospholipid-Antikörper sowie die Gerinnungsparameter waren unauffällig. Die Thrombozytenzahl war unter Therapie mit Anagrelid mit $291 \times 10^{9} / 1$ im Normbereich.

\section{Ergänzende Untersuchungen}

Die Doppler- und Duplex-Untersuchungen des oberflächlichen und tiefen Beinvenensystems zeigten keine Mündungsklappeninsuffizienz oder Hinweise auf das Vorliegen einer chronisch-venösen Insuffizienz. Die Pulse an den Füßen und Leisten waren gut tastbar und bei normwertigen brachiotibialen Indizes (BTI) konnte eine pAVK ausgeschlossen werden. Eine Polyneuropathie war klinisch nicht festzustellen. Die bakteriologische und mykologische Diagnostik war unauffällig. Die histopathologischen Untersuchung einer aus dem Wundrand entnommenen Biopsie zeigte eine ausgeprägte fibrosierende Dermatitis ohne Hinweis auf eine floride Vaskulitis.

\section{Therapie und Verlauf}

Nach initialem chirurgischen Debridement führten wir ein modernes feuchtes Wundmanagement unter Verwendung von u. a. Hydrokolloidverbänden, Biochirurgie und Vakuumversiegelung über 3 Wochen ohne wesentliche Befundverbesserung durch. In Absprache mit den Kollegen aus der Hämatologie setzten wir Anagrelid ab. Unter engmaschigen Blutbildkontrollen stieg jedoch innerhalb einer Woche die Thrombozytenzahl auf pathologische Werte, sodass interdiszipilinär entschieden wurde, erneut eine Therapie mit Hydroxyurea zu beginnen. Im weiteren Verlauf heilten die Ulcera crurum unter Fortführung der konservativen Lokaltherapie mit Verwendung einer Vakuumversiegelung und modernen Wundverbänden innerhalb von 8 Wochen vollständig ab ( $\odot$ Abb. 1 c, d).

Nach Ausschluss anderer Ursachen stellten wir die Diagnose von Ulcera crurum in Zusammenhang mit der Einnahme von Anagrelid.
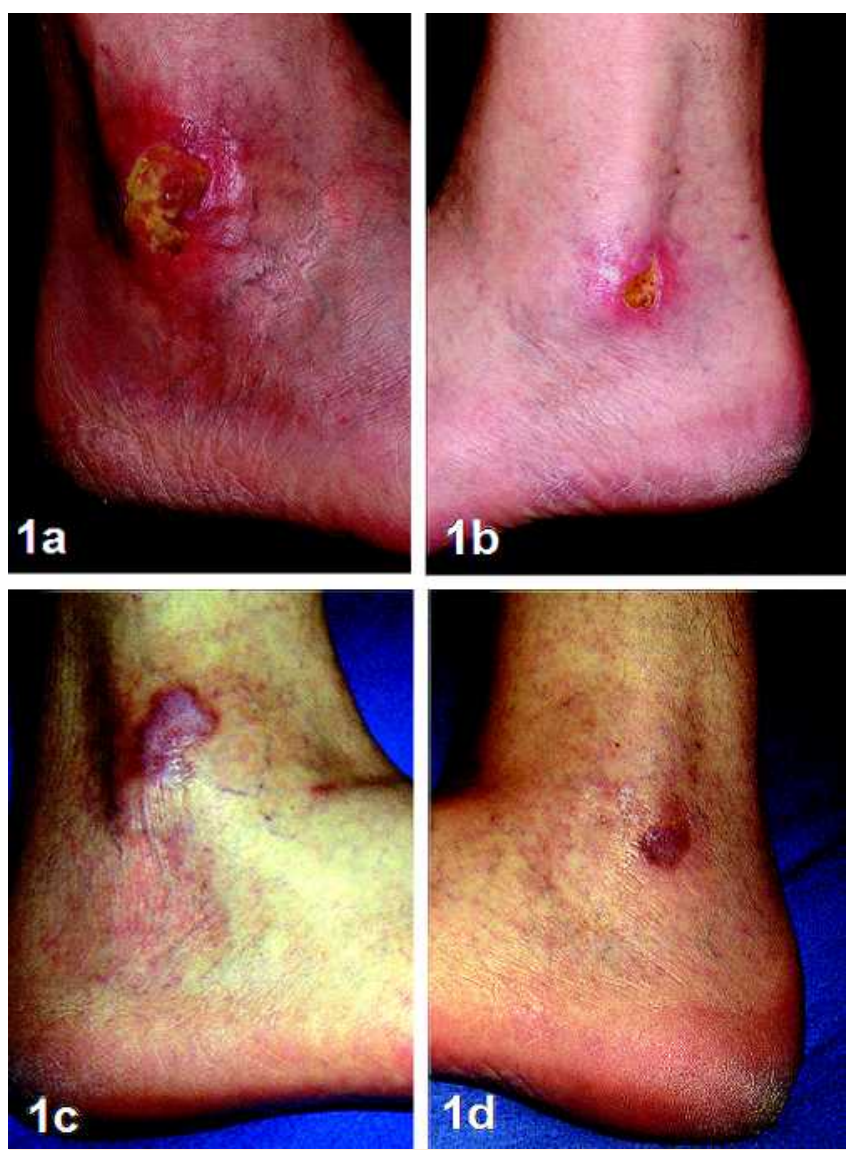

Abb. 1 a Ulcus cruris am rechten Malleolus lateralis nach chirurgischem Debridement. $\mathbf{b}$ Fibrinös belegtes Ulcus cruris am linken Malleolus lateralis. $\mathbf{c}$ und $\mathbf{d}$ Vollständig abgeheilte Ulcera crurum 8 Wochen nach Absetzen von Anagrelid.

\section{Diskussion}

In der Literatur wurden Ulzerationen als Nebenwirkung verschiedener Medikamente wie beispielsweise Marcumar, Nifedipin oder Hydroxyurea bereits mehrfach beschrieben $[6,10,13]$. Wir konnten jedoch keine Publikation über das erstmalige Auftreten von Ulcera crurum unter Einnahme von Anagrelid finden. Es existiert lediglich ein Fallbericht über Ulzerationen unterTherapie mit Anagrelid, bei denen es sich um ein Rezidiv eines zuvor bereits abgeheilten Hydroxyurea-induzierten Ulcus cruris handelte [9].

Anagrelid ist ein Imidazoquinazolin, das 2005 in Deutschland u.a. für die Behandlung der essenziellen Thrombozythämie zugelassen wurde. Die hemmende Wirkung von Anagrelid auf die humane Thrombozytenbildung wird über eine Verzögerung der Megakaryozyten-Reifung durch Inhibition der zyklischen AMPPhosphodiesterase III vermittelt. Kanzerogene, insbesondere Leukämie-induzierende Effekte konnten bislang nicht nachgewiesen werden. Die am häufigsten beschriebenen Nebenwirkungen sind Kopfschmerzen, Durchfall, Palpitationen, Schwächegefühl, Schmerzen, Benommenheit, Bauchschmerzen und Übelkeit. Die überwiegende Zahl der Nebenwirkungen bildet sich jedoch innerhalb von 2-3 Wochen zurück $[4,8]$. Hydroxyurea hingegen ist ein hydroxyliertes Harnstoffderivat, das für zahlreiche kutane Nebenwirkungen wie beispielsweise Nagelverfärbungen, Dermatomyositis-artigen Hautveränderungen, Alopezie und Ulzerationen bekannt ist $[3,11,12]$. Die meist äu- 
ßerst schmerzhaften Ulzerationen treten nach langjähriger Einnahme von Hydroxyurea insbesondere symmetrisch an den Malleolen, Fersen oder auch den Fußrücken auf [7]. In der Literatur wird das Auftreten dieser Ulzerationen meist 1-10 Jahre nach Beginn der Therapie beschrieben $[3,12]$. Die Pathogenese konnte bislang nicht eindeutig geklärt werden. Es wurde eine Graft-versus-Host-ähnliche Reaktion mit Degeneration der basalen Keratinozyten und einer epidermodermalen Spaltbildung vermutet [3]. Ebenso wurde ein zytostatischer Effekt auf die basalen Keratinozyten oder eine Störung der Mikrozirkulation durch Effekte auf Erythrozyten mit Verminderung der Anzahl, Zunahme des mittleren Volumens und somit verminderte Verformbarkeit diskutiert [11,14].

In dem hier beschriebenen Fallbericht entwickelten sich die Ulcera crurum 6 Wochen nach Absetzen von Hydroxyurea und Beginn der Therapie mit Anagrelid. Somit könnten sie entweder als Folge der Langzeiteinnahme von Hydroxyurea oder als Ausdruck der neu eingeleiteten Therapie mit Anagrelid gesehen werden. Aufgrund der Tatsache, dass die Ulcera crurum trotz optimierter Lokaltherapie unter Fortführung der Behandlung mit Anagrelid persistierten, jedoch nach Umsetzen der Therapie auf die erneute Einnahme von Hydroxyurea bei Fortführung der konservativen Therapie vollständig abheilten, sehen wir den kausalen $\mathrm{Zu}$ sammenhang am ehesten durch die Einnahme von Anagrelid bedingt. Der genaue Pathomechanismus, der letztendlich für das Entstehen der vermutlich durch Anagrelid induzierten Ulcera crurum verantwortlich sein könnte, bleibt weiterhin unbekannt.

\section{Konsequenz für Klinik und Praxis}

Schmerzhafte, symmetrisch auftretende Ulcera crurum im Bereich der Malleolen sollten differenzialdiagnostisch auch an Anagrelid als ursächlichen Faktor denken lassen.

Neben einer modernen feuchten Wundtherapie ist die Umsetzung der Therapie mit Anagrelid für die Abheilung der Ulzerationen notwendig.

Autorenerklärung: Die Autoren erklären, dass sie keine finanziellen Verbindungen mit einer Firma haben, deren Produkt mit dem Beitrag eine wichtige Rolle spielt (oder mit einer Firma, die ein Konkurrenzprodukt vertreibt).

\section{Abstract}

\section{Leg Ulcers Associated with Intake of Anagrelide $\nabla$}

Anamnesis and clinical findings: Extremely painful leg ulcers on the lateral aspect of both ankles developed in a 38-year-old man for the first time six weeks after starting treatment with anagrelide for thrombocythemia.

Investigations: Extensive diagnostic tests, including laboratory tests, Doppler and duplex ultrasound of the superficial and deep leg veins, histopathology and bacteriological and mycological tests excluded vascular disease, vasculitis and metabolic causes. Therapy and course: Despite adequate local treatment the ulcerations did not improve until anagrelide was discontinued and replaced by hydroxyurea.

Conclusion: Anagrelide administration was the most likely cause of the leg ulcerations, even though this possible side effect has only been described once in the literature.

\section{Literatur}

1 Dissemond J. Ulcus cruris - Grundlagen, Diagnostik und Therapie, 1. Auflage UNI-MED Verlag, Bremen, London, Boston, 2005

2 Dissemond J, Höft D, Knab J, Franckson T, Kröger K, Goos M. Leg ulcer in a patient associated with hydroxyurea therapy. Int J Dermatol 2006; 2: $158-160$

3 Eming SA, Peters T, Hartmann $K$ et al. Lichenoid chronic graft-versushost-disease-like acrodermatitis induced by Hydroxyurea. J Am Acad Dermatol 2001; 45: $321-323$

4 Harrison CN, Campbell PJ, Buck G et al. United Kingdom Medical Research Council Primary Thrombocythemia 1 Study. Hydroxyurea compared with anagrelide in high-risk essential thrombocythemia. N Engl J Med 2005; 7: $33-45$

5 Körber A, Dissemond J. Aktuelle Therapieoptionen des Ulcus cruris venosum Teil I. Wundforum 2005; 3: $8-12$

6 Luca S, Romeo S. Edema and skin ulcers of the lower limbs as a collateral effect of nifedipine: A clinical case report. Minerva Cardioangiol 1999; 476: 219-222

7 Montefusco E, Alimenta G, Gastaldi R et al. Unusal dermatologic toxicity of long term therapy with hydroxyurea in chronic myelogenous leukaemia. Tumori 1986; 72: 317-322

8 Petrides PE, Trapp O, Beykirch MK. Anagrelid (Agrelin), pharmakologisches Profil und klinische Anwendung. Onkologe 1997; 3: 298 - 302

9 Ruiz-Arguelles GJ, Ruiz-Delgado GJ, Ruiz-Reyes G, Chernoff SG. Anagrelide-induced relaps of a hydroxyurea-induced leg ulcer in a patient with primary thrombocythemia. Mayo Clin Proc 1998; 11: 1125-1125

10 Schmidt M, Sitter T, Spannagl M, Schramm W, Held E. Hautnekrose im Vorfußbereich unter Antikoagulation mit Phenprocoumon. Dtsch Med Wochenschr 1999; 124: 727-730

11 Sirieix ME, Debure C, Baudot $N$ et al. Leg ulcers and Hydroxyurea - Forty-one cases. Arch Dermatol 1999; 135: 818-820

12 Weber L, Schick E, Merkel $M$ et al. Dermatomyositisartige Hautveränderungen unter Hydroxyurea (Litalir ${ }^{\circledR}$ )-Langzeittherapie. Hautarzt 1995; 46: 717-721

13 Weinlich G, Fritsch P. Leg ulcers in patients treated with hydroxyurea for myeloproliferative disorders: what is the trigger: A clinical case report. Br J Dermatol 1999; 7: 171 - 172

14 Weinlich G, Schuler G, Greil R et al. Leg ulcers associated with longterm hydroxyurea therapie. J Am Acad Dermatol 1998; 8: 372 - 374

15 Welt K, Ott S, Weiss JM, Scharffetter-Kochanek K. Schmerzhafte symmetrische Knöchelulzerationen. Hautarzt 2004; 55: 190-193 